\title{
Clinical Study \\ Factor V-Leiden Mutation: A Common Risk Factor for Venous Thrombosis among Lebanese Patients
}

\author{
Raghid Kreidy \\ Department of Vascular Surgery, Saint George Hospital, University Medical Center, University of Balamand, \\ P.O. Box 166378, Achrafieh, Beirut 1100 2807, Lebanon
}

Correspondence should be addressed to Raghid Kreidy, docrkdy@inco.com.lb

Received 22 January 2012; Revised 17 March 2012; Accepted 9 April 2012

Academic Editor: Solaf Elsayed

Copyright (c) 2012 Raghid Kreidy. This is an open access article distributed under the Creative Commons Attribution License, which permits unrestricted use, distribution, and reproduction in any medium, provided the original work is properly cited.

\begin{abstract}
Aim. Lebanon exhibits one of the highest prevalences of factor V-Leiden (FVL) in the world (14.4\%). The aim of this study is to evaluate the incidence of FVL mutation among Lebanese patients with lower extremity venous thrombosis. Material and Methods. From January 2003 to January 2011, 283 consecutive Lebanese patients, diagnosed with deep venous thrombosis (DVT) by duplex scan, were retrospectively reviewed. FVL mutation was tested among patients with conditions highly suggestive of hypercoagulation states (65 patients). Results. FVL mutation was detected among $56.9 \%$ of patients, $68.6 \%$ of patients younger than 50 years, and $43.4 \%$ of patients older than 50 years $(P=0.041)$. FVL mutation was commonly reported in young adults, in patients with pregnancy, estrogen drugs, recurrent DVT, and resistance to anticoagulation. Conclusion. The high rate of FVL mutation observed among Lebanese patients with venous thrombosis is related to the high prevalence of this mutation in the Lebanese population. Thrombophilia screening should be tailored to accommodate a population's risk factor. In countries with high prevalence of FVL, this mutation should be screened among patients younger than 50 years and patients with situations highly suggestive of hypercoagulation states.
\end{abstract}

\section{Introduction}

The appropriate management of venous thrombosis requires a thorough knowledge of diagnostic and treatment modalities. However, an understanding of the underlying epidemiology and associated risk factors is equally essential. Pathophysiology of venous thrombosis is multi-factorial and involves environmental, acquired, and genetic factors. Factor V R 506 Q-Leiden is the most frequent prothrombotic genetic abnormality leading to thrombophilia. The prevalence rate of this mutation varies according to ethnic and geographic distribution of the populations. The allele mutation is high among Eastern Mediterranean populations and extremely high among Lebanese population. The aim of this study is to determine the incidence of factor V-Leiden mutation among Lebanese patients with lower extremity venous thrombosis and high risk situations for hypercoagulation states and to discuss the reported results according to epidemiologic and pathophysiologic publications in the international literature.

\section{Material and Methods}

From January 2003 to January 2010, 283 consecutive Lebanese patients (115 males and 168 females), diagnosed in an academic tertiary-care center with lower extremity deep venous thrombosis using color flow duplex scan (Prosound Alpha 7 ALOKA, Zug, Switzerland), were retrospectively reviewed. Age varied between 21 to 96 years (mean: 64.9 years). Factor V-Leiden was screened among 65 of the 283 patients, presenting with specific conditions highly suggestive of hypercoagulation states according to the guidelines proposed by the European Genetics Foundation, the Cardiovascular Educational and Research Trust, the International Union of Angiology, and the Mediterranean League on Thromboembolism [1]. These conditions were young age, pregnancy, oral contraceptives and estroprogestative drugs, spontaneous, extended and recurrent DVT, family history, resistance to anticoagulation, long-haul air travel, and constituted the inclusion criteria for testing FVL. 
There was no exclusion criteria. Accordingly, FVL mutation was tested in 37 young patients, in 9 pregnant patients, in 5 patients using oral contraceptives or treated with hormones, in 32 patients with spontaneous venous thrombosis, in 9 patients with extended venous thrombosis, in 15 patients with recurrent venous thrombosis, in 11 patients with family history of venous thromboembolism (VTE), in 4 patients with resistance to anticoagulants, and in 2 patients with longhaul air travel. Fourteen patients presented with one of these high risk conditions requiring FVL testing, 18 with two, 2 with three and 3 with four conditions.

Inherited prothrombotic risk factors including FVL, prothrombin G 20210 A, factor V H 1299 R, MTHFR C $677 \mathrm{~T}$, and MTHFR A $1298 \mathrm{C}$ mutations were determined using CVD strips according to the protocol supplied by the manufacturer (CVD Strip Assays R, ViennaLab, Austria). Plasma homocysteine level was also reported.

Patients were subdivided into 2 groups according to age-A: younger than 50 years (35 patients) and B: older than 50 years (30 patients).

A clinical research form was filled out with every patient, by a retrospective evaluation of clinical presentation and risk factors for venous thrombosis; data were entered and analysed by SPSS Statistics (IBM Corporation, Somers, NY, USA) software, version 13.0. A chi-square test was used to correlate between dichotomous variables, and a Fisher exact test was used in case of calculated values that were lower than five.

\section{Results}

Factor V-Leiden was detected among 37 patients representing $56.9 \%$ of the tested patients. More than one clinical risk factor was associated with a higher risk of positivity for FVL (63\% comparing to $44 \%$ for the other prothrombotic genetic mutations). Five patients $(7.7 \%)$ were carriers for the homozygous form and thirty-two $(49.2 \%)$ for the heterozygous form. Factor V-Leiden was isolated in 19 patients and associated with prothrombin G 20210 A mutation in 4 patients and with other prothrombotic genetic mutations in 14 patients (MTHFR C 677 T mutation: homozygote: 2, heterozygote: 9, MTHFR A 1298 C mutation: heterozygote: 8, and V H 1299 R mutation: heterozygote: 1) (Table 1). Plasma homocysteine level was increased in 8 patients. The association with other genetic risks did not increase the risk of recurrent DVT but was determinant of more severe and extended forms of VTE. Factor V-Leiden was more commonly reported in the younger age group 9 (group A) comparing to the older age group (group B) $(68.6 \%$ comparing to $43.4 \% ; P=0.041$ ).

FVL mutation was detected among $66.6 \%$ of pregnant patients, $60 \%$ of patients using oral contraceptives or treated with hormone therapy, $56.2 \%$ of patients with spontaneous DVT, $55.5 \%$ with extended DVT, $66.6 \%$ of patients with recurrent DVT, $45.4 \%$ of patients with family history of venous thromboembolism, $100 \%$ of patients with resistance to anticoagulants and $50 \%$ of patients with long-haul air travel (Table 2).
TABle 1: Prothrombotic genetic mutations associated with FVL mutation.

\begin{tabular}{lc}
\hline Prothrombotic genetic mutation & Number of patients \\
\hline Prothrombin G 20210 A & 4 \\
MTHFR C 677 T & 11 \\
MTHFR A 1298 C & 8 \\
V H 1299 R & 1 \\
\hline
\end{tabular}

TABLe 2: Conditions associated with FVL mutation.

\begin{tabular}{lcc}
\hline High risk conditions & $\begin{array}{c}\text { Patients } \\
\text { screened for } \\
\text { FVL mutation }\end{array}$ & $\begin{array}{c}\text { Patients carriers for } \\
\text { FVL mutation }\end{array}$ \\
\hline Young patients (A group) & 35 & $24(68.6 \%)$ \\
Pregnancy & 9 & $6(66.6 \%)$ \\
Estrogen treatment & 5 & $3(60 \%)$ \\
Spontaneous DVT & 32 & $18(56.2 \%)$ \\
Extended DVT & 9 & $5(55.5 \%)$ \\
Recurrent DVT & 15 & $10(66.6 \%)$ \\
Family history of VTE & 11 & $5(45.4 \%)$ \\
Resistance to anticoagulant & 4 & $4(100 \%)$ \\
Long-haul air travel & 2 & $1(50 \%)$ \\
\hline
\end{tabular}

\section{Discussion}

Resistance to activated protein $\mathrm{C}$ degradation caused by a mutation in the factor V R 506 Q-Leiden is the most prevalent inherited cause of venous thrombosis. The prevalence rate of factor $\mathrm{V}$-Leiden in the general population varies from $0 \%$ to $15 \%$ according to ethnicity and geographic distribution [2, 3]. The allele mutation is low in African, Asian, South European populations (1\%-3\%), relatively high in United States populations (5\%) and high in Mediterranean populations $(13.6 \%$ in Syria, $12.3 \%$ in Jordan, and $13.4 \%$ in Greece) $[4,5]$. Lebanon exhibits one of the highest frequencies of FVL mutation in the Eastern Mediterranean and in the world with a prevalence of $14.4 \%$ in the general population [6].

Among the inherited thrombophilias, factor V-Leiden gene mutation is the most common predisposing factor, accounting for $10 \%$ to $20 \%$ of VTE in large population studies $[7,8]$. Some authors suggest that this mutation is present in up to $37 \%$ of patients with postthrombotic syndrome [1]. The lifetime probability of symptomatic VTE in patients with heterozygous FVL mutation is approximately $10 \%$ [9]. The risk of VTE is increased 3- to 7-fold in heterozygotes for FVL and 50- to 100-fold in homozygotes $[8,9]$.

The prevalence of FVL mutation among patients with venous thrombosis varies considerably according to the origin of the reported study. It is extremely low in Singapore $(0.26 \%)$, low in Spain and in the United States $(8.3 \%$ and 9\%, resp.), relatively high in the United Kingdom, Greece, and Tunisia $(15.6 \%, 16.8 \%$, and $20.3 \%$, resp.) and high in Italy $(24.3 \%)$ [10-15]. This prevalence significantly increases among adults younger than 50 years of age in Denmark 
(32.7\%) and Czech Republic (40\%), but remains very low in India (3\%) [16-18]. Children developing venous thrombosis are often carriers for FVL mutation. Unal et al. suggested that homozygosity for FVL leads to the development of thrombosis at a young age [19]. Aschka et al. demonstrated that $26 \%$ of children less than 5 years of age and $30 \%$ of children more than 10 years of age with venous thrombosis and $29 \%$ of children with spontaneous venous thrombosis have harbored FVL mutation [20]. In these series, the high rate of factor $\mathrm{V}$-Leiden mutation observed among the tested patients $(56.9 \%)$ and the tested young adults (group A) (68.6\%) is related to the high prevalence of this mutation in the Lebanese population. The mutant allele was highly expressed among Lebanese patients with DVT and high risk conditions for hypercoagulation states. These findings confirm that FVL mutation is a very common risk factor for venous thrombosis among Lebanese patients and especially young Lebanese adults with high risk situations, emphasizing the importance of screening for this mutation in this particular group of patients.

VTE recurrence rate increases significantly in adults and children when venous thrombosis is associated with primary hypercoagulopathy and essentially with factor V-Leiden mutation $[21,22]$. More than $66 \%$ of the reported patients with recurrence were carriers for FVL mutation.

FVL mutation, especially in its homozygote form, increases the risk for venous thrombosis 34-fold among pregnant women and 35-fold among contraceptive users $[13,23-25]$. FVL mutation has been identified in $33.3 \%$ of pregnant women and $6.5 \%$ of oral contraceptive users women $[26,27]$. In these series, a high percentage of pregnant women $(66.6 \%)$ and of women treated with estrogen drugs $(60 \%)$ were carriers for FVL mutation. Screening for FVL mutation among Lebanese pregnant women and among women treated with estrogen and presenting for venous thrombosis is warranted.

A very high incidence of FVL mutation was particularly detected among patients manifesting resistance to anticoagulants (100\%). Testing for FVL among Lebanese patients is highly recommended when venous thrombosis is extensive and progressive despite adequate anticoagulation.

FVL mutation interacts with other concurrent acquired and thrombophilic conditions such as cancer, surgery, longhaul air travel, and with associated prothrombotic genetic abnormalities and essentially prothrombin G 20210A mutation to increase the risk of incident venous thrombosis [9, 28-33]. The relative risk for venous thrombosis is calculated to be 2-3-fold for the prothrombin mutation alone and 20-fold for a combination with FVL mutation [34]. The three reported patients harboring this combination were young adults and two of them presented for extensive and bilateral VTE. FVL acts also as a concurrent risk factor in individuals with other prothrombotic polymorphism and hyperhomocysteinemia leading to a synergistic gene-gene interaction often increasing the risk above the sum of individual risk factors [35].

In these series, FVL mutation was associated with MTHFR C 677 T mutation in 9 patients, MTHFR A 1298
C mutation in 8 patients and VH $1299 \mathrm{R}$ mutation in 1 patient. Of the six reported patients carriers for two or more prothrombotic genetic defects in addition to FVL mutation and presenting increased homocysteine level, four patients manifested bilateral extended DVT with pulmonary embolism resistant to anticoagulants and requiring inferior vena cava filter insertion.

Duration of treatment for venous thrombosis among patients carriers for FVL mutation depends on the nature and extension of the thrombosis, the underlying risk factors, the presence of a previous VTE, and the presence and the severity of concurrent prothrombotic genetic mutation. Patients homozygotes for FVL mutation and double heterozygotes for FVL and prothrombin mutation are considered for extended long-term treatment with anticoagulants therapy [36].

\section{Conclusion}

The high rate of factor $\mathrm{V}$-Leiden mutation observed among Lebanese patients with lower extremity venous thrombosis is related to the high prevalence of this mutation among the Lebanese population. This factor interacts with other concurrent acquired and thrombophilic conditions to increase the risk of incident venous thrombosis.

The prevalence of FVL mutation is particularly increased among young patients, pregnant patients, patients treated with estrogen, patients with recurrent DVT, and patients with resistance to anticoagulation. The association of two or more prothrombotic genetic defects to FVL mutation further increases the risks and the severity of venous thrombosis.

Thrombophilia screening should be tailored to accommodate a population's risk factor. In countries with high prevalence of factor V-Leiden, this mutation should be tested among patients younger than 50 years even with a transient risk factor and patients with situations highly suggestive of hypercoagulation states. This permits to extend the duration of anticoagulant therapy in high risk patients reducing the incidence of postthrombotic syndrome and recurrent venous thromboembolism.

\section{References}

[1] A. N. Nicolaides, H. K. Breddin, P. Carpentier et al., "Thrombophilia and venous thromboembolism. International consensus statement. Guidelines according to scientific evidence," International Angiology, vol. 24, no. 1, pp. 1-26, 2005.

[2] M. Montagnana, E. J. Favaloro, M. Franchini, G. C. Guidi, and G. Lippi, "The role of ethnicity, age and gender in venous thromboembolism," Journal of Thrombosis and Thrombolysis, vol. 29, no. 4, pp. 489-496, 2010.

[3] L. N. Roberts, R. K. Patel, and R. Arya, "Venous thromboembolism and ethnicity," British Journal of Haematology, vol. 146, no. 4, pp. 369-383, 2009.

[4] W. H. Geerts, J. A. Heit, G. P. Clagett et al., "Prevention of venous thromboembolism," Chest, vol. 119, no. 1, pp. S132S175, 2001.

[5] D. C. Rees, M. Cox, and J. B. Clegg, "World distribution of factor V Leiden," Lancet, vol. 346, no. 8983, pp. 1133-1134, 1995. 
[6] N. Irani-Hakime, H. Tamim, R. Kreidy, and W. Y. Almawi, "Prevalence of factor V R506 Q-Leiden among apparently healthy Lebanese patients," American Journal of Hematology, vol. 65, no. 1, pp. 45-49, 2000.

[7] S. Moll, "Thrombophilias_practical implications and testing caveats," Journal of Thrombosis and Thrombolysis, vol. 21, no. 1, pp. 7-15, 2006.

[8] M. A. Crowther and J. G. Kelton, "Congenital thrombophilic states associated with venous thrombosis: a qualitative overview and proposed classification system," Annals of Internal Medicine, vol. 138, no. 2, pp. 128-134, 2003.

[9] G. A. F. Nicolaes and B. Dahlback, "Activated protein C resistance (FVL) and thrombosis: factor $\mathrm{V}$ mutations causing hypercoagulable states," Hematology/Oncology Clinics of North America, vol. 17, no. 11, pp. 37-61, 2003.

[10] L. H. Lee, T. C. Liu, P. Kuperan, L. K. Tan, D. Tan, and M. L. Poon, "Hereditary thrombophilia in an unselected cohort of venous thrombosis patients in Singapore," Journal of Clinical Pathology, vol. 64, no. 9, pp. 814-817, 2011.

[11] V. Roldan, R. Lecumberri, J. F. S. Muñoz-Torrero et al., "Thrombophilia testing in patients with venous thromboembolism. Findings from the RIETE registry," Thrombosis Research, vol. 124, no. 2, pp. 174-177, 2009.

[12] J. A. Friedline, E. Ahmad, D. Garcia et al., "Combined factor $\mathrm{V}$ Leiden and prothrombin genotyping in patients presenting with thromboembolic episodes," Archives of Pathology and Laboratory Medicine, vol. 125, no. 1, pp. 105-111, 2001.

[13] O. Wu, L. Robertson, S. Twaddle et al., "Screening for thrombophilia in high-risk situations: systematic review and costeffectiveness analysis. The Thrombosis: Risk and Economic Assessment of Thrombophilia Screening (TREATS) study," Health Technology Assessment, vol. 10, no. 11, pp. 1-75, 2006.

[14] A. Hatzaki, E. Anagnostopoulou, V. Metaxa-Mariatou et al., "The impact of heterozygosity for the factor $\mathrm{V}$ Leiden and factor II G20210A mutations on the risk of thrombosis in Greek patients," International Angiology, vol. 22, no. 1, pp. 7982, 2003.

[15] A. Ajem, A. Slama, F. B. H. Slama, and T. Mehjoub, "Prevalence of factor V Leiden mutation in patients with thrombosis in Tunisia," Eastern Mediterranean Health Journal, vol. 15, no. 6, pp. 1483-1488, 2009.

[16] B. S. Andersen and J. Olsen, "Oral contraception and factor $\mathrm{V}$ Leiden mutation in relation to localization of deep vein thrombosis," Thrombosis Research, vol. 90, no. 4, pp. 191-194, 1998.

[17] P. Dulíček, J. Malý, L. Pešavová, and M. Pecka, "Prevalence of inherited thrombophilia in young thrombosis patients from the East Bohemian region," Blood Coagulation and Fibrinolysis, vol. 13, no. 6, pp. 569-573, 2002.

[18] K. Ghosh, S. Shetty, M. Madkaikar et al., "Venous thromboembolism in young patients from Western India: a study," Clinical and Applied Thrombosis/Hemostasis, vol. 7, no. 2, pp. 158-165, 2001.

[19] S. Unal, G. Balta, F. Duru, F. Gumruk, C. Altay, and A. Gurgey, "Clinical and laboratory evaluation of Turkish children with thrombosis for homozygous factor V G1691A mutation," Blood Coagulation and Fibrinolysis, vol. 15, no. 4, pp. 343-346, 2004.

[20] I. Aschka, V. Aumann, F. Bergmann et al., "Prevalence of factor $\mathrm{V}$ leiden in children with thrombo-embolism," European Journal of Pediatrics, vol. 155, no. 12, pp. 1009-1014, 1996.

[21] A. Marchiori, L. Mosena, M. H. Prins, and P. Prandoni, "The risk of recurrent venous thromboembolism among heterozygous carriers of factor $\mathrm{V}$ Leiden or prothrombin
G20210A mutation. A systematic review of prospective studies," Haematologica, vol. 92, no. 8, pp. 1107-1114, 2007.

[22] U. Nowak-Göttl, R. Junker, W. Kreuz et al., "Risk of recurrent venous thrombosis in children with combined prothrombotic risk factors," Blood, vol. 97, no. 4, pp. 858-862, 2001.

[23] E. R. Pomp, A. M. Lenselink, F. R. Rosendaal, and C. J. M. Doggen, "Pregnancy, the postpartum period and prothrombotic defects: risk of venous thrombosis in the MEGA study," Journal of Thrombosis and Haemostasis, vol. 6, no. 4, pp. 632637, 2008.

[24] C. Legnani, B. Cosmi, L. Valdrè et al., "Venous thromboembolism, oral contraceptives and high prothrombin levels," $J$ Thromb Haemost, vol. 1, no. 1, pp. 112-117, 2003.

[25] J. P. Vandenbroucke, T. Koster, E. Briet, P. H. Reitsma, R. M. Bertina, and F. R. Rosendaal, "Increased risk of venous thrombosis in oral-contraceptive users who are carriers of factor V Leiden mutation," Lancet, vol. 344, no. 8935, pp. 1453-1457, 1994.

[26] M. Procházka, V. Krcová, P. Hrachovec, M. Kudela, and L. Slavík, "Activated protein $\mathrm{C}$ resistance and deep venous thrombosis in pregnancy," Ceska Gynekologie, vol. 67, no. 5, pp. 251-254, 2002.

[27] J. Paseka, V. Unzeitig, D. Cibula, A. Bulikova, M. Matykova, and K. Chroust, "Factor V Leiden in oral contraceptives users," Ceska Gynekologie, vol. 65, no. 3, pp. 156-159, 2000.

[28] J. W. Blom, C. J. M. Doggen, S. Osanto, and F. R. Rosendaal, "Malignancies, prothrombotic mutations, and the risk of venous thrombosis," Journal of the American Medical Association, vol. 293, no. 6, pp. 715-722, 2005.

[29] P. Prandoni, "Cancer and thromboembolic disease: how important is the risk of thrombosis?" Cancer Treatment Reviews, vol. 28, no. 3, pp. 133-136, 2002.

[30] M. Mandalà, S. Barni, M. Prins et al., "Acquired and inherited risk factors for developing venous thromboembolism in cancer patients receiving adjuvant chemotherapy: a prospective trial," Advances in Gastrointestinal Cancers, vol. 21, no. 4, pp. 871-879, 2009.

[31] W. H. Geerts, G. F. Pineo, J. A. Heit et al., "Prevention of venous thromboembolism: the 7th ACCP conference on antithrombotic and thrombolytic therapy," Chest, vol. 126, no. 3, supplement, pp. 338S-400S, 2004.

[32] W. Schobersberger, B. Schobersberger, and H. Partsch, "Travel-related thromboembolism: mechanisms and avoidance," Expert Review of Cardiovascular Therapy, vol. 7, no. 12, pp. 1559-1567, 2009.

[33] J. Siedenburg, "Traveler's thrombosis. Who is at risk-low to prevent it," Münchener Medizinische Wochenschrift, vol. 144, no. 38, pp. 39-41, 2002.

[34] S. R. Poort, F. R. Rosendaal, P. H. Reitsma, and R. M. Bertina, "A common genetic variation in the 3'-untranslated region of the prothrombin gene is associated with elevated plasma prothrombin levels and an increase in venous thrombosis," Blood, vol. 88, no. 10, pp. 3698-3703, 1996.

[35] V. De Stefano, P. Chiusolo, K. Paciaroni, and G. Leone, "Epidemiology of factor V Leiden: clinical implications," Seminars in Thrombosis and Hemostasis, vol. 24, no. 4, pp. 367379, 1998.

[36] G. Palareti, "Current criteria to determine the duration of anticoagulant therapy," Recenti Progressi in Medicina, vol. 98, no. 12, pp. 603-606, 2007. 


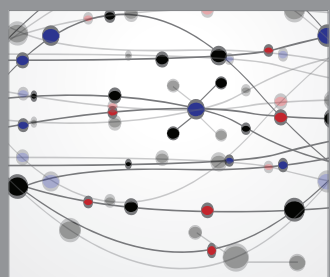

The Scientific World Journal
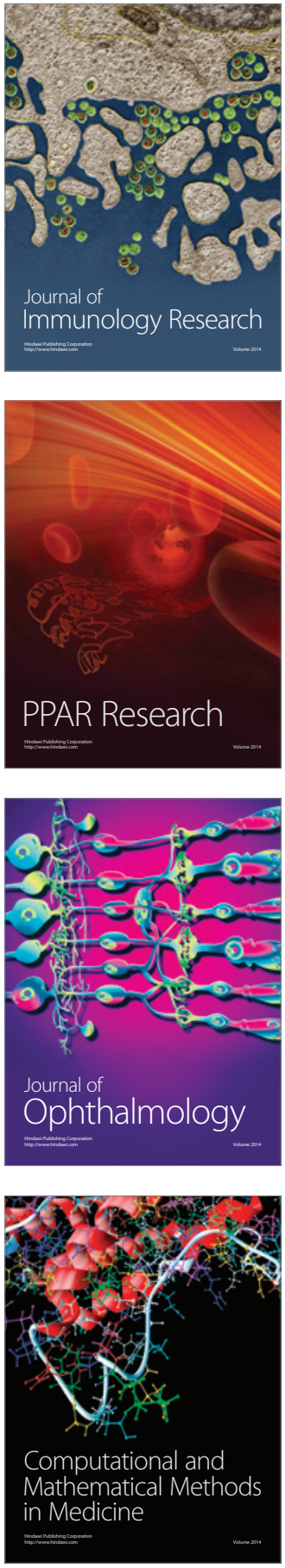

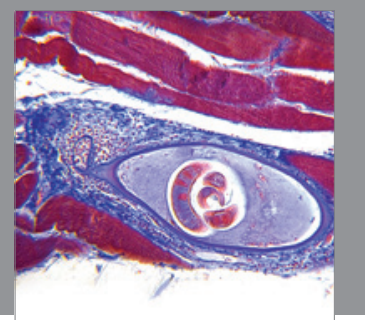

Gastroenterology

Research and Practice
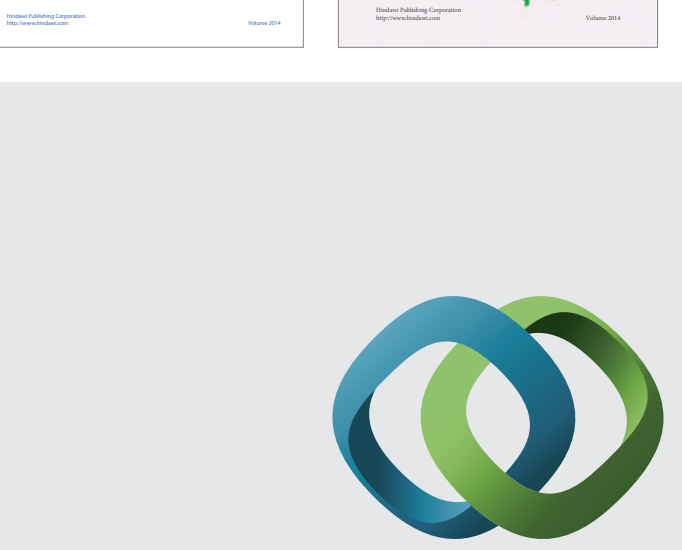

\section{Hindawi}

Submit your manuscripts at

http://www.hindawi.com
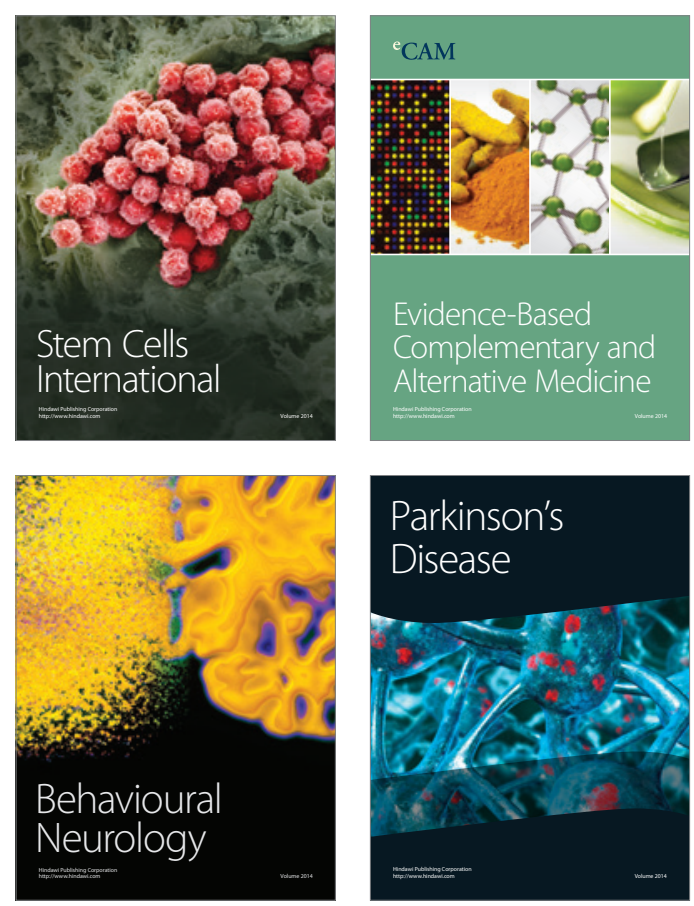

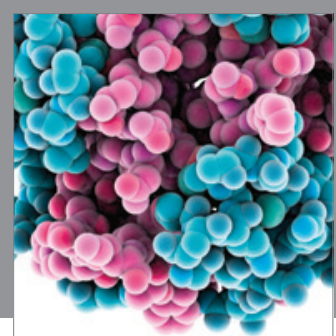

Journal of
Diabetes Research

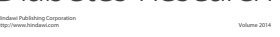

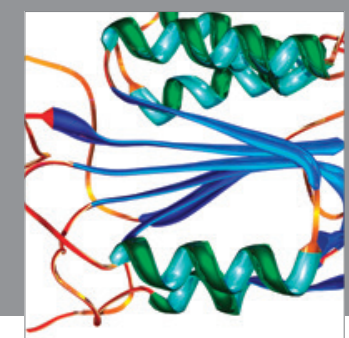

Disease Markers
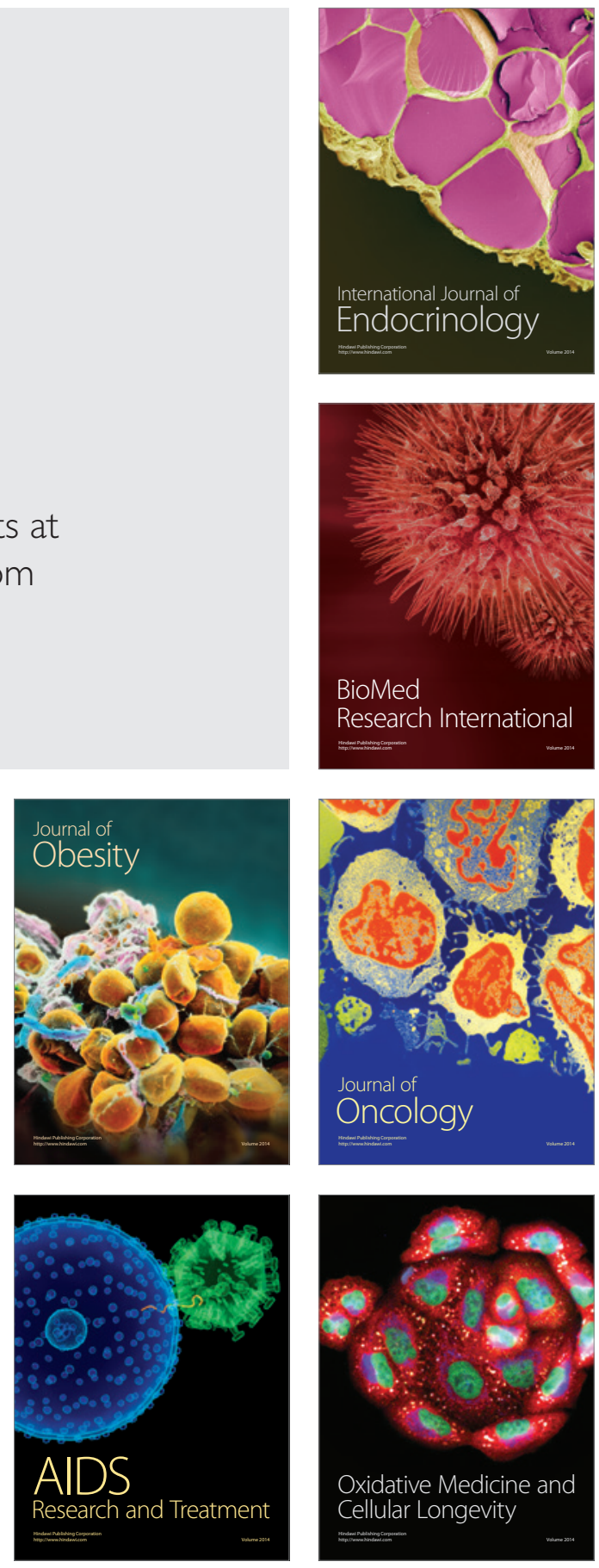